\title{
Densidad poblacional y algunos aspectos de la ecología del carrá (Huberodendron patinoi Cuatrec.) en la zona alta y media del río Baudó, Chocó, Colombia
}

\section{Population density and some aspects of carrá ecology (Huberodendron patinoi Cuatrec.) in the high and average area of the Baudó river, Chocó, Colombia}

\author{
Haidyn Luis Moreno Mosquera*
}

\section{Resumen}

Para evaluar la densidad de individuos del carrá (Huberodendron patinoi) en la zona del Alto y Medio Baudó se utilizó un muestreo diagnóstico, donde se establecieron 197 parcelas de $10 \times 10 \mathrm{~m}$. Se registró un total de 423 individuos de la especie de los cuales 12 fueron caracterizados como árboles semilleros localizados en las comunidades de Bellavista Bubasa, puerto Echeverri y puerto Araceli. Con respecto al estado de desarrollo se reportaron 134 brizales, 47 latizales y 242 fustales. Las clases diamétricas I, II, y VII fueron las más representativas con 181, 80 y 56 individuos respectivamente. Del total de árboles registrados tan solo 39 presentaron malformación fenotípica. Brosimun utile y Dictiocarion platiceparum fueron las especies que mayoritariamente se encontraran asociadas con el carrá. La densidad poblacional calculada a partir del área de muestreo reportó un total de 215 individuos por hectárea, datos que dan cuenta de la presencia de la especie, lo que permitió concluir que al menos para esta zona del Baudó no se identificaron índices de agotamiento de la misma.

Palabras clave: Carrá, Clases diamétricas, Densidad población.

\begin{abstract}
To evaluate the density of carrá (Huberodendron patinoi) individuals in the Alto and Medio Baudó area, a diagnostic sampling was used, in which 197 plots of $10 \times 10 \mathrm{~m}$ were established. A total of 423 individuals of the species were recorded, of which 12 were characterized as seed trees located in the communities of Bellavista Bubasa, Echeverri port and Araceli port. Regarding the state of development, 134 brizales, 47 latizales and $242 \mathrm{fus}$
\end{abstract}

* Ingeniero agroforestal e Investigador tipo I, Instituto de Investigciones Ambientales del Pacífico (IIAP), Quibdó, Colombia. e-mail: $\underline{\text { hmoreno@iiap.org.co }}$ 


\section{Bioetnia Volumen 13, 2016}

tales were reported. Diametric classes I, II, and VII were the most representative with 181, 80, and 56 individuals respectively. Of the total number of registered trees, only 39 had phenotypic malformation. Brosimun utile, and Dictiocarion platiceparum were the species that were mostly associated with carrá. The population density calculated from the sampling area reported a total of 215 individuals per hectare, data that account for the presence of the species, which allowed to conclude that at least for this area of Baudó no indices of depletion were identified.

Keywords: Carrá, Diametric classes, Population density.

\section{Introducción}

El Chocó Biogeográfico a pesar de contar con una gran riqueza, se enfrenta a una problemática ambiental, social y económica en las distintas áreas de bosques naturales que se traduce en su deterioro progresivo y en la pérdida significativa de biodiversidad, esto como producto de la creciente realización de actividades productivas ilegales como la minería, extracción indiscriminada de especies forestal y cultivos ilícitos, que amenazan y deterioran aceleradamente las condiciones de los ecosistemas.

Actualmente se estima que más del $40 \%$ de la producción de madera en Colombia proviene de fuentes ilegales (BM 2006). El proceso de deforestación en el país se está produciendo principalmente en las tierras bajas del Pacífico, que contiene $8 \%$ de los bosques naturales del país y la mitad de su territorio está cubierta por bosques, sobre todo en selvas (García 2012). La deforestación está asociada con la minería, la tala insostenible, la infraestructura en desarrollo y la expansión agrícola.

En el caso de las plantas, si bien muchas especies se encuentran actualmente amenazadas en el país, es evidente la presión directa a la que son so- metidas las especies maderables, porque incluso su demanda mundial promueve su aprovechamiento. En Colombia existen 34 especies maderables en alguna categoría de amenaza, encontrándose 15 especies en peligro, 10 en peligro crítico y 9 en vulnerable (Cárdenas y Salinas 2007). La mayoría de especies están ubicadas en el departamento del Chocó, donde se han registrado 13 especies maderables amenazadas, tales como Humiriastrum procerum (chanó), Huberodendrum patinoi (carrá), Mora oleifera (nato) y Prioria copaifera (cativo) (Cárdenas y Salinas 2007).

Huberodendron patinoi. A nivel nacional se encuentra en la categoría vulnerable (VU A2cd). (Cárdenas y Salinas 2007). Con este trabajo se confirma esta categoría y se agrega e lumbral «a» para VU A2acd, porque gran parte de sus poblaciones se han reducido por la intensa explotación maderera a que es sometida la especie, lo que se evidencia en la cantidad de tocones que se encontraron. Se estima que $35 \%$ de su población ha sido diezmada. De esta especie sólo se hallan algunos individuos maduros de las poblaciones históricas remanentes por estar ubicados en el $\mathrm{CMUCH}$ donde existe un poco restringida la extracción maderera (Rengifo-Ibargüen 2010). La situación antes mencionada hace necesario adelantar el presente estudio el cual tiene por objetivo evaluar la densidad poblacional y otros aspectos del carrá en el municipio de Medio y Alto Baudó

\section{Metodología}

Área de estudio. El municipio del Alto Baudó tiene una extensión total de $1.532 \mathrm{~km}^{2}$; se enmarca en la cuenca del río Baudó y como contexto geográfico se ubica en el centro y sur del departamento del Chocó, limitando al norte con los municipios de Quibdó, Bojayá y Bahía Solano; al oriente con los municipios de Quibdó, Río Quito y Cantón de San Pablo; al occidente con el municipio de Nuquí; al sur con los municipios de Bajo Baudó 


\section{Carrá en zona alta y media del río Baudó. HL Moreno Mosquera}

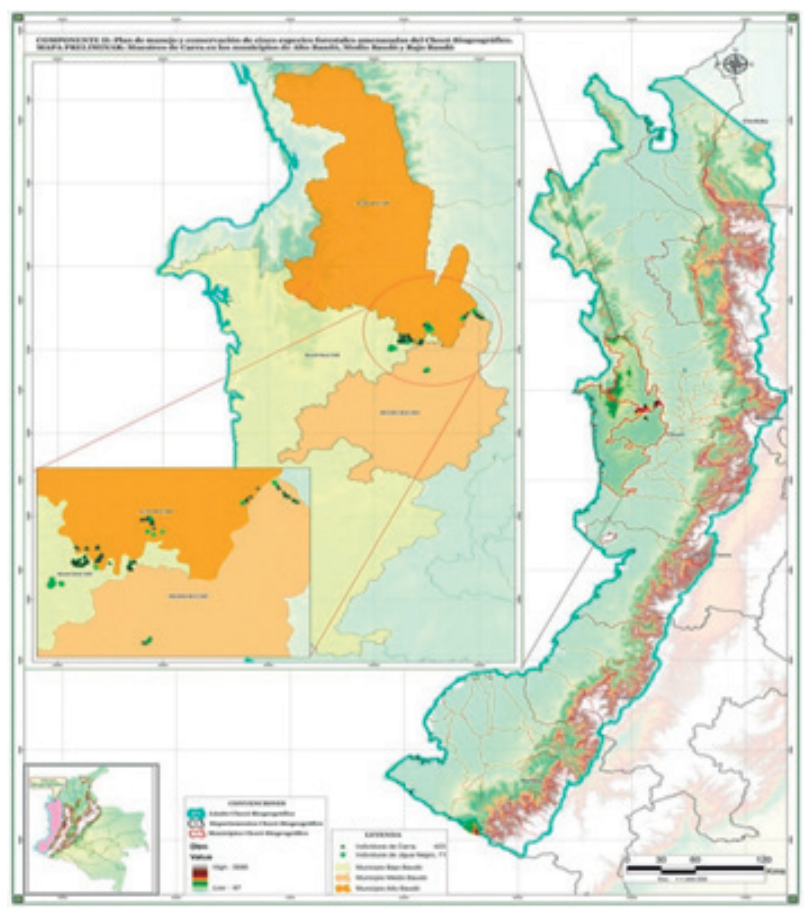

Figura 1. Ubicación geográfica de la zona de estudio, municipios de Alto y Medio Baudó.

y Medio Baudó, su cabecera Pie de Pató. Está localizada en la margen izquierda del río Baudó, a los $05^{\circ} 31^{\prime} 33^{\prime \prime}$ de latitud norte y $76^{\circ} 59^{\prime} 42^{\prime \prime}$ de longitud oeste (Figura 1). Su altura sobre el nivel del mar es de $50 \mathrm{~m}$, temperatura media de $28^{\circ} \mathrm{C}$, precipitación media anual de $6.439 \mathrm{~mm}$ y dista de Quibdó $80 \mathrm{~km}$.

Municipio de Medio Baudó. Tiene una extensión total de $4.840 \mathrm{~km}^{2}$. Limita por el norte con el municipio de Alto Baudó, por el sur con el municipio de Bajo Baudó, por el occidente con el municipio de Bajo Baudó y al oriente con Istmina y Cantón de San Pablo. Su cabecera municipal es Puerto Meluk que se encuentra a $12 \mathrm{msnm}$, con una temperatura media de $28^{\circ} \mathrm{C}$.

Método. Para la toma de información primaria se utilizó el muestreo diagnóstico, haciendo recorridos de rastreo de la especie; una vez ubicados los individuos, fueron delimitadas y establecidas con cuerdas de polietileno, 197 parcelas cuadradas de $10 \mathrm{~m}$ x10 m a lo largo de diferentes microambientes (colinas, pendientes, planicies y bosques de influencia hídrica) obte-
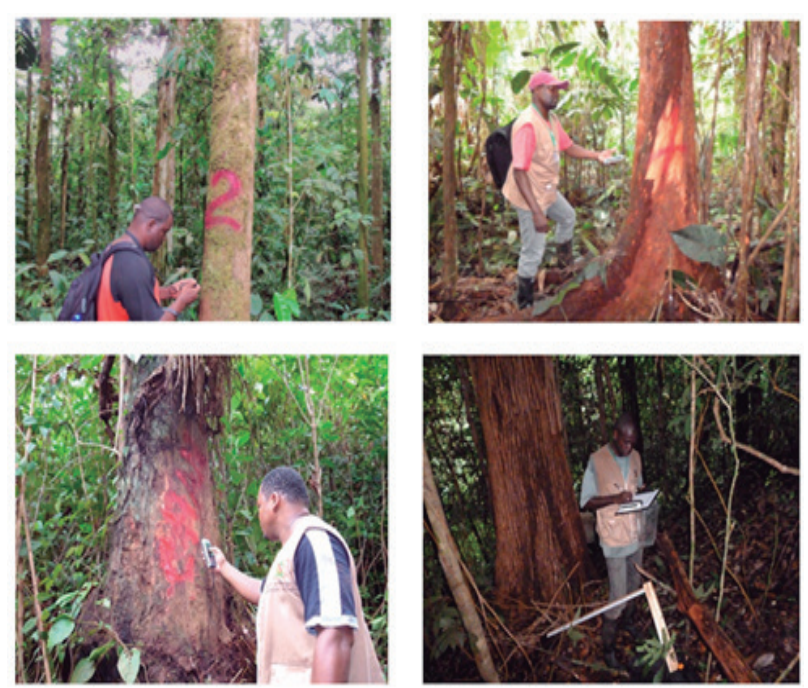

Figura 2. Registro y marcaje de los individuos en campo.

niendo un área total de muestreo de 1,97 hectáreas, en los municipios de Alto y Medio Baudó. En cada parcela se registraron y midieron todos los individuos de la especie objeto de estudio, incluyendo todos los estados de desarrollo (brinzal, latizal y fustal) (Figura 2). Cada 20 parcelas establecidas, se registraron datos de vegetación asociada con las especies.

La determinación del número de individuos de la especie que ocurren por unidad de área (ind/ $\mathrm{m}^{2}$ ), se realizó mediante el calculó la densidad. Para registrar el diámetro a la altura del pecho de los individuos, se utilizó una forcípula, la cual fue ubicada aproximadamente a $1,30 \mathrm{~m}$ sobre el nivel del suelo. Además, se calculó la altura de los árboles (m), definido desde la distancia del suelo a su punta o ápice. Adicionalmente, se registró la altura comercial, considerando la medida desde el DAP hasta donde inicia la ramificación principal de los árboles. Por otro lado, el análisis de estados de desarrollo se evaluó considerando los siguientes rangos: brinzal: individuos que van desde 0 a $5 \mathrm{~cm}$ de diámetro; latizal: individuos que van desde 5 a $10 \mathrm{~cm}$ de diámetro y fustal: individuos mayores de $10 \mathrm{~cm}$ de diámetro. Finalmente, para evaluar el crecimiento fustal de los individuos se registraron las clases diamétricas, las cuales se 
dividieron en siete categorías, así: I= individuos con DAP entre 0-9 cm; II: DAP entre 10-20 cm; III: DAP entre 21-30 cm; IV: DAP entre 31-40 $\mathrm{cm}$; V: DAP entre $41-50 \mathrm{~cm}$; VI: DAP entre 51$60 \mathrm{~cm}$ y VII: categoría en la que se ubicaron los individuos con ADP mayores a $60 \mathrm{~cm}$.

Además de la información anterior se consideraron algunos aspectos de la ecología de las especies como calidad del fuste, presencia deárbol semillero y especies asociadas. De igual manera se obtuvieron colecciones de hojas y frutos de la especie en las parcelas que sirvieron de base para asegurar la adecuada identificación de los individuos; adicional a lo anterior, se tomaron registros fotográficos, se marcaron los arboles con aerosol y por último se georeferenciaron con la ayuda de un equipo satelital GPS. Toda la información de las especies por localidad se registró en fichas prediseñadas que incorpora información dasométricas de la especie las cuales se tabularon en bases de datos en Excel 2007.

\section{Resultados y discusiones}

Densidad por hectárea muestreada. En total se registraron 423 individuos de carrá en un área de 1,97 ha, lo que equivale a una densidad poblacional de $0,02 \mathrm{ind} / \mathrm{m}^{2}$ equivalente a 215 individuos por hectárea (Figura 3), densidad que se puede explicar a la poca extracción forestal que se realiza en la zona, porque la alternativas económicas de las comunidades se orientan a otro tipo de actividades productivas como cultivos de uso ilícitos, tala de otras especies y la agricultura (siembra de cultivo de plátano, yuca, maíz entre otros). Estos datos son consecuentes con los resultados obtenidos por CODECHOCÓ (2010) en el plan de ordenación de la cuenca alta del río Baudó, en el que se identificaron 249 individuos de H. patinoi. Murillo (2009) reporta 87 individuos de la especie H. patinoi. Sin embargo, contrastan con otros estudios realizados en el departamento (IIAP y CODECHOCO 2008) en los que se registran 7 y 8 árboles para carrá en los municipios de Riosucio y Carmen de Darién, hecho que se asocia con la alta vocación forestal de las comunidades de esta parte del Chocó que tienen la extracción maderera como su principal actividad de ingreso económico.

Estado de desarrollo de carrá en los sitios de muestreo. Del total de individuos inventariados, $57 \%$ (242) corresponde a fustales, $32 \%$ (134) a brinzales y tan solo $11 \%$ (47) a latizales (Figura $3)$. Se observa que el fustal muestra una marcada diferencia de individuos en relación con los otros estados de desarrollo, pues más del $50 \%$ de los árboles pertenecen a este estado. La especie también muestra una diferencia marcada entre los dos estados inferiores, por un lado, el estado de brinzal reporta 134 individuos lo que demuestra la presencia de procesos de regeneración natural de la especie, no obstante, se observa una significativa reducción de individuos en el estadío siguiente (latizales 47 ind), hecho que podría estar asociado

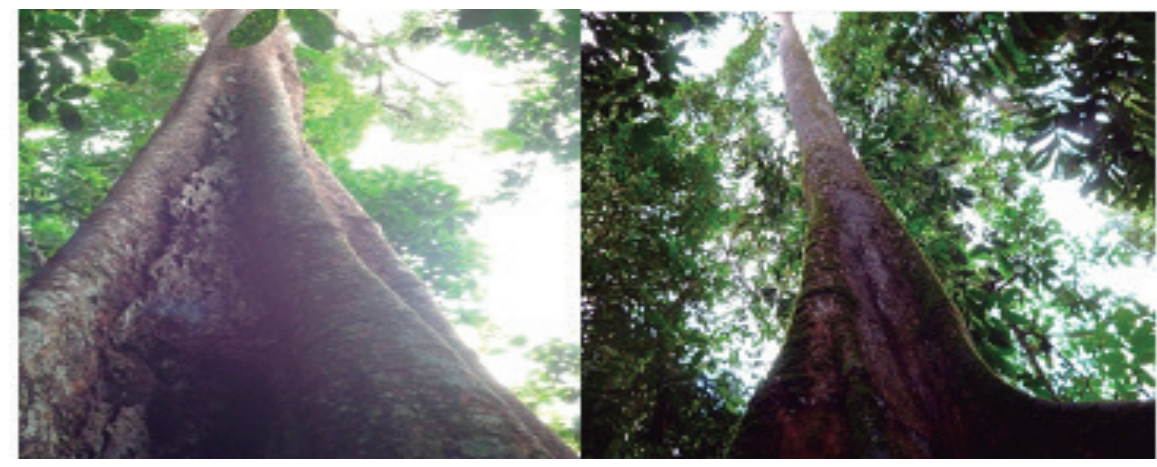

Figura 3. Árboles maduros de carrá registrados en la zona de muestreo. 


\section{Carrá en zona alta y media del río Baudó. HL Moreno Mosquera}

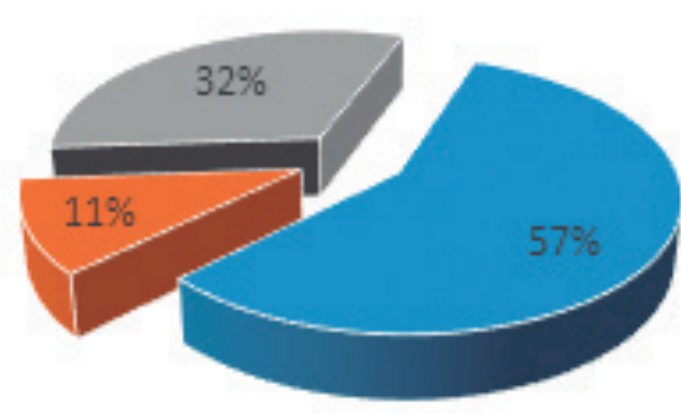

- Fustal " Latizal "Brinzal

Figura 4. Distribución porcentual por categoría de desarrollo de los individuos de carrá registrados en el área de estudio.

con la dinámica del bosque y con las limitaciones y competencia por luz en algunos estratos. Sin embargo, la estructura poblacional observada en cada estado de desarrollo, sugiere la persistencia de la especies en el área, además, el hecho de que el mayor porcentaje de individuos registrado corresponda a la categoría de desarrollo de fustal, demuestra la sobrevivencia de los mismo a las categorías anteriores, lo que indica que la mayor mortalidad de los individuos se presentan en el estado sucesiones medio, hecho que puede estar relacionarse con dinámicas poblacionales como la competencia intra e inter específica por espacio y/o nutrientes (Figuras 4 y 5).
Distribución de la especie por clases diamétricas. Más del $60 \%$ de los individuos de $H$. patinoi registrados correspondieron a las clases diamétricas I-II con el $42,8 \%$ y $18,9 \%$ respectivamente, mientras que $13,2 \%$ de los individuos se ubicaron en la clase diamétrica VII, a la que corresponden los individuos que presentaron los mayores DAP (Figura 6). El hecho de que $62 \%$ de los individuos se encuentre en las clases diamétricas inferiores, evidencia el buen estado poblaciones y capacidad de regeneración natural de la especie en el área estudiada. Además, el registro de individuos en las clases superiores muestra la persistencia y sostenimiento de sus poblaciones, pues de acuerdo con Guariguata (1998), la poca existencia de individuos en los rangos diamétricos inferiores afecta la regeneración a la vez que incrementa el riesgo a la extinción.

Según Palacios y Ramos (1999) citado por Mosquera et al. (2007), un aspecto que influye mucho en la presencia de diámetros pequeños o medianos en los bosques del Chocó, es la presencia de muchos claros naturales ocasionados por la caída de uno o varios árboles, provocada por la acción de fuertes vientos, deslizamiento del terreno o por la dinámica natural del bosque, al igual que por la alta precipitación y densidad, condiciones que fueron observadas en el área de estudio. Esto contrasta con lo expresado por
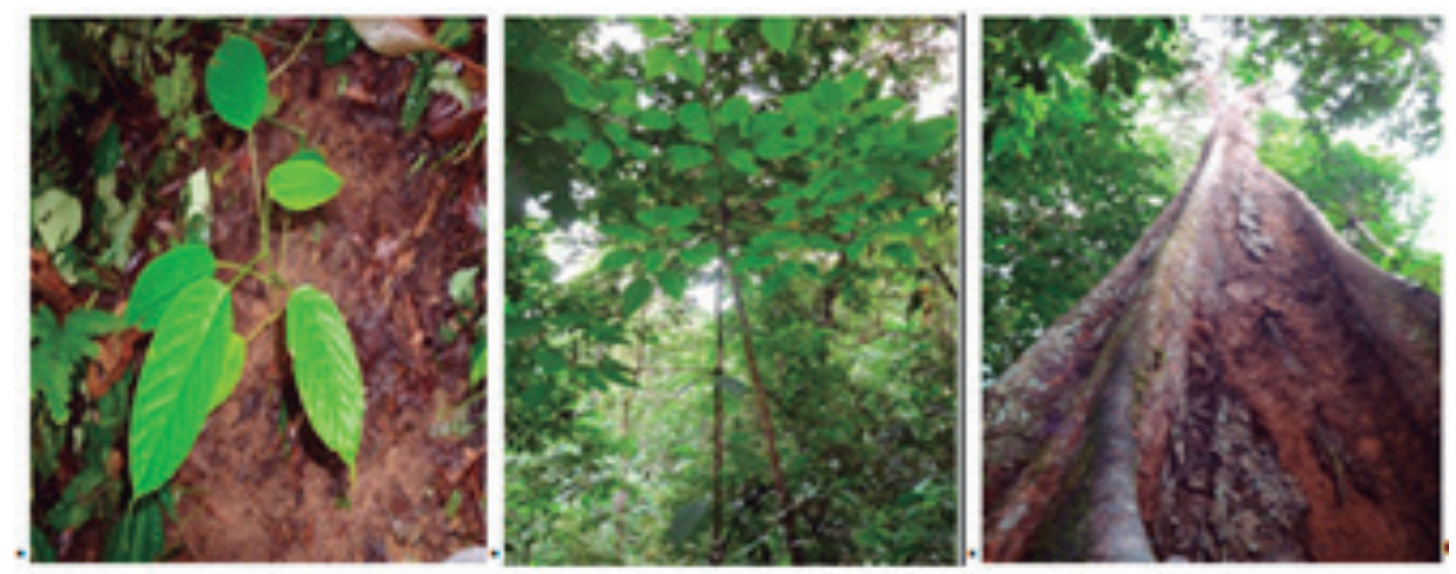

Figura 5. Estado de desarrollo de los individuos de carrá registrados en el área de estudio. A. Brinzal, B. Latizal, C. Fustal. 
Bioetnia Volumen 13, 2016

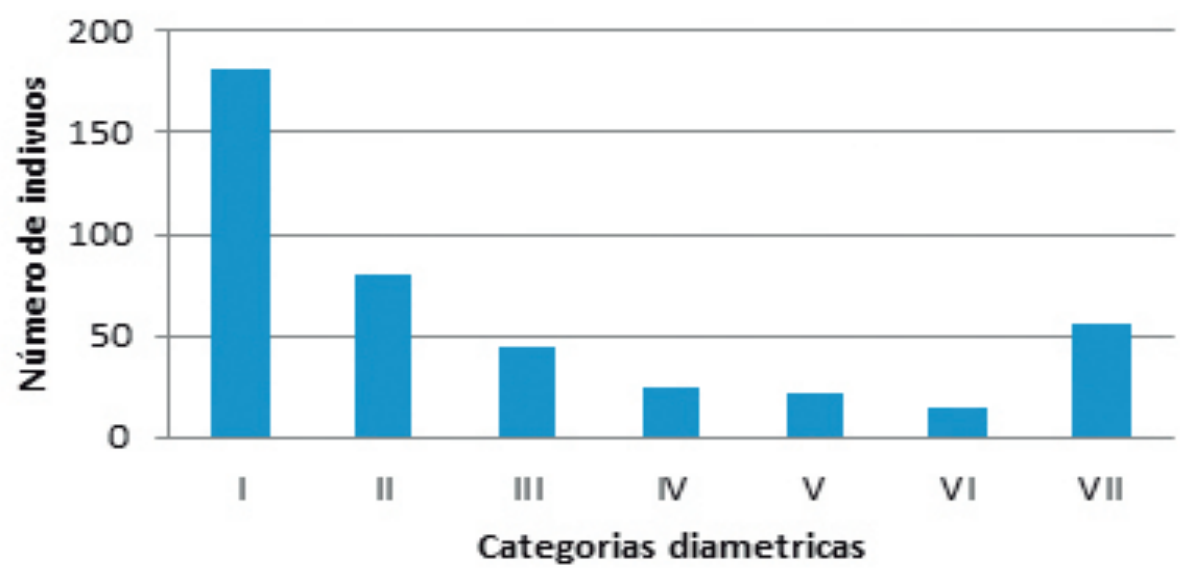

Figura 6. Distribución de las clases diamétricas de los individuos de H. patinoi, registrados en el Alto y Medio Baudó.

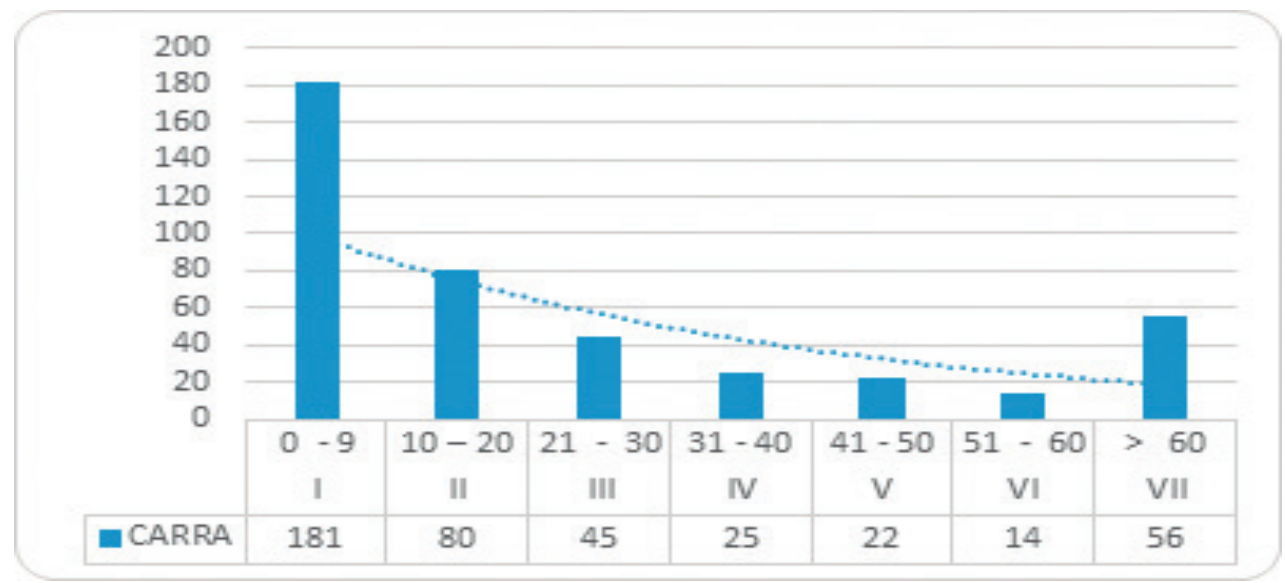

Figura 7. Distribución de la especie por clase diamétrica.

Lamprecht (1990) quien manifiesta que la distribución diamétrica de los individuos en bosques nativos jóvenes o en procesos de recuperación, presenta una tendencia en forma de "J" invertida (Figura 7).

Presencia de árboles semilleros. De los 70 árboles de $H$. patinoi con diámetro superior a 50 $\mathrm{cm}$, solo 17\% (12 individuos), fueron identificados como árboles semilleros pues fue observado a su alrededor procesos de regeneración natural, situación común en especies dioicas por cuanto las flores masculinas y femeninas se encuentran en plantas separadas y por consiguiente, sólo una parte de la población (los árboles con flores femeninas) formarán semillas y facilitaran la regeneración (Figura 8, Tabla 1 ).

Calidad fenotípica del fuste. Las observaciones de las características del tronco y las ramas que tienen importancia para la calidad, muestran que del total de individuos registrados, se identificaron 39 con algún grado de malformación, de los cuales 36 presentaron bifurcación del tronco y 3 se encontraban partidos, el porcentaje es superior $9,2 \%$ lo que permite afirmar que la especie muestra un buen comportamiento en estado natural con respecto al tema. Sin embargo, la mayor parte de los individuos de fustales de $H$. patino presentaron bambas en la base de la raíz lo cual corrobora que es una característica propia de la especie (Figura 9). 


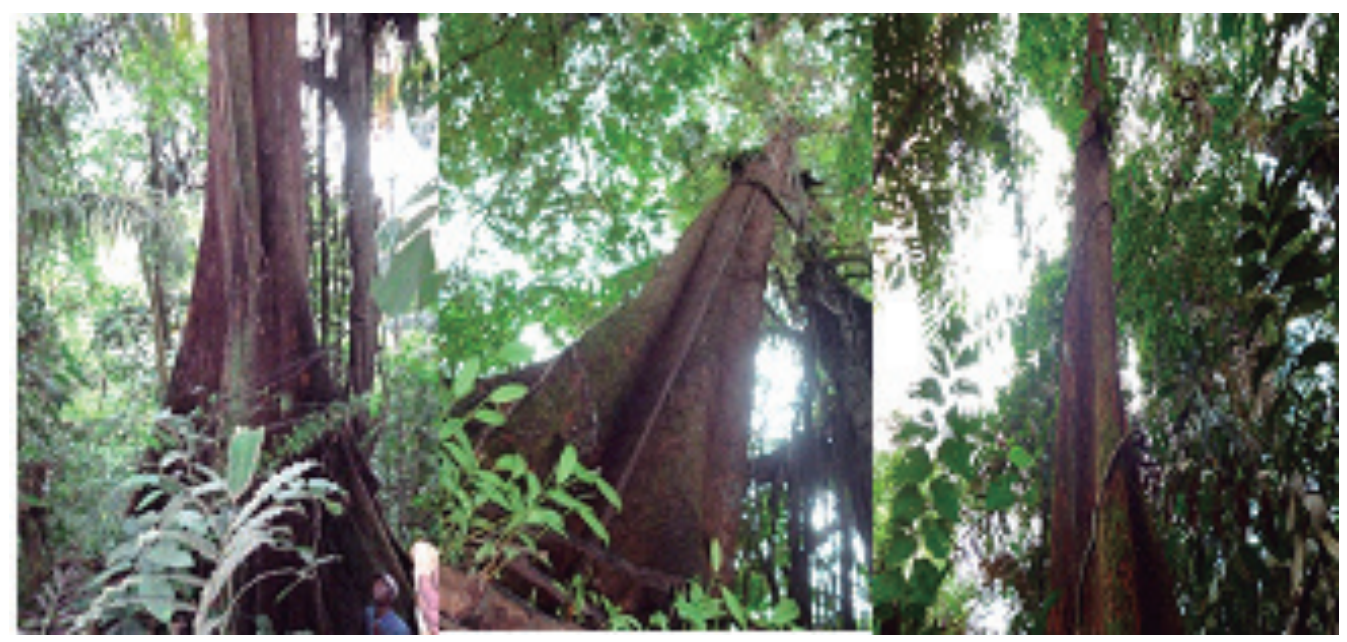

Figura 8. Árboles semilleros de carrá encontrados en la zona de muestreo.

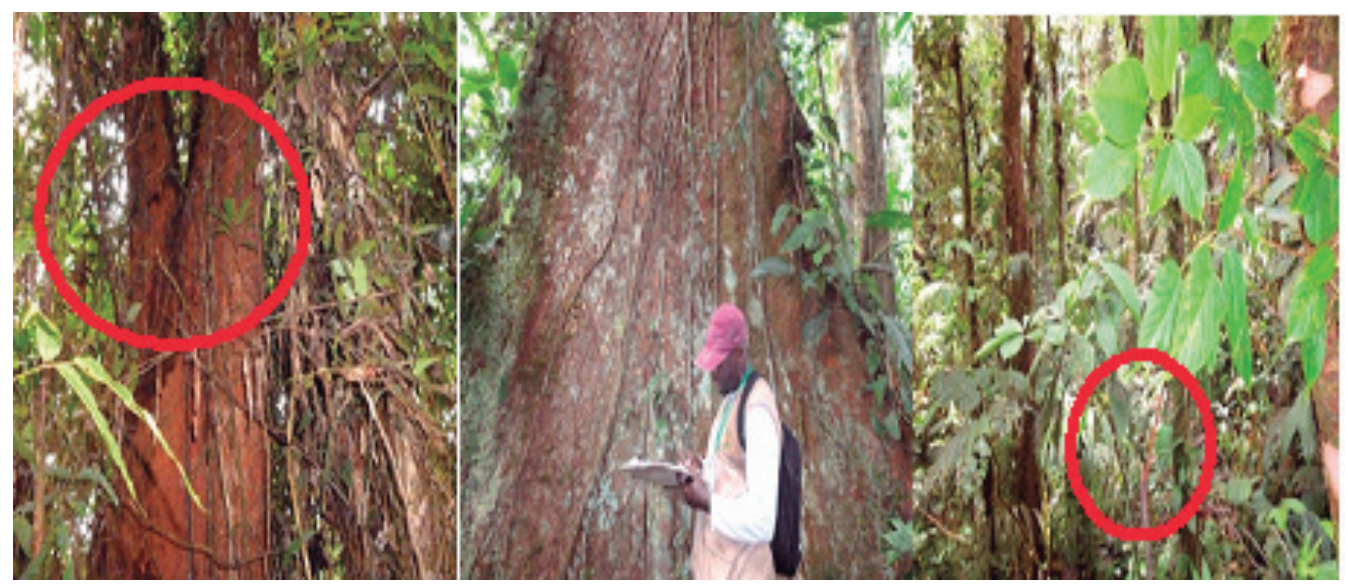

Imágenes 9. Registros de calidad del fuste. A. Bifurcado, B. Bambas de raíz, C. Partido.

Especies forestales asociadas con carrá. En total se registraron 18 especies forestales asociadas con carrá. Las que más se repitieron en las parcelas fueron el lechero Brosimun utile y la palma barrigona Dictiocarion platiceparu que se reconocieron en 7 y 4 de las 9 parcelas en donde se realizaron los muestreos para evaluar las especies asociadas; caucho (Castilloa elastica Sesé, C) y lechero (Brosimum utile) representan la familia morácea que junto con la mimosáceas como el guamo (Inga sp) y aserrín (Parkia pendula) son las de mayor prevalencia de todas la reportadas. La Tabla 3 muestra la identificación de las especies maderables asociadas con la especie de interés reportadas en el área de estudio.

\section{Conclusiones y recomendaciones}

En relación con el método utilizado y el área muestreada, se observa una alta densidad de individuos por hectárea, lo que resulta relevante si se consideran los reportes de reducción de la especie en otros municipios del departamento y del país para presentarse como una potencial área de conservación para las especies.

La especie muestra claros signos de buena regeneración natural y adecuado desarrollo de individuos en los estados de brinzal y fustal, lo que sugiere el potencial de persistencia y sostenibilidad de la especie en la región. Sin embargo, se requiere avanzar en evaluaciones 


\section{Bioetnia Volumen 13, 2016}

Tabla 1. Ubicación geográfica de los árboles semilleros de carrá referenciados en el área de estudio

\begin{tabular}{|c|c|c|}
\hline \multirow{2}{*}{ Comunidad } & \multicolumn{2}{|c|}{ Coordenadas } \\
\hline & $x$ & $\mathbf{Y}$ \\
\hline \multirow[t]{3}{*}{ Puerto Echeverri } & $05^{\circ} 17^{\prime} 42,9^{\prime \prime}$ & $076^{\circ} 58$ '43,8” \\
\hline & $05^{\circ} 18^{\prime} 25,8^{\prime \prime}$ & $077^{\circ} 00^{\circ} 04,1^{\prime \prime}$ \\
\hline & $05^{\circ} 18,1^{\prime} 19,4 "$ & $077^{\circ} 00$ ' 11,3 ” \\
\hline \multirow[t]{5}{*}{ Bellavista Dubasa } & $05^{\circ} 18^{\prime} 23,3 "$ & $077^{\circ} 00$ ‘04,9” \\
\hline & $05^{\circ} 18^{\prime} 22,8^{\prime \prime}$ & $077^{\circ} 00^{\circ} 07,7^{\prime \prime}$ \\
\hline & $05^{\circ} 18^{\prime} 17,1^{\prime \prime}$ & $077^{\circ} 00^{\circ} 08,8^{\prime \prime}$ \\
\hline & $05^{\circ} 18,1^{\prime} 19,2 "$ & $077^{\circ} 00$ '09,4” \\
\hline & $05^{\circ} 18,00,3 "$ & $077^{\circ} 01$ '23,2” \\
\hline \multirow[t]{4}{*}{ Puerto Araceli } & $05^{\circ} 17, ' 58,4 "$ & $077^{\circ} 01$ '50,6” \\
\hline & $05^{\circ} 17, ' 51,9 "$ & $077^{\circ} 00$ ‘56,7” \\
\hline & $05^{\circ} 18,{ }^{\prime} 03,3 "$ & $077^{\circ} 01$ ' 13,3 " \\
\hline & $05^{\circ} 18, ' 01,2 "$ & $077^{\circ} 00$ '45,3" \\
\hline
\end{tabular}

silviculturales (apertura de claros y/o cierre de dosel) para determinar los factores que influyen en la reducción tan significativa de los individuos en estado de latizal.

Las clases diamétricas I y II representan la mayor cantidad de individuos registrados en los muestreos realizados siguiendo en orden de importancia por la categoría VII que representa a los mayores de $60 \mathrm{~cm}$. Esta es de gran importancia para la sostenibilidad ecológica de la especie porque se evidencia un buen comportamiento de los procesos de regeneración y poca presión antrópica en el estado fustal.

La identificación de 12 árboles semillero en la zona es un aspecto importante, por lo que se recomienda el diseño de planes de seguimiento, conservación y manejo, porque estos son fuente para obtener semillas que permitan adelantar programas de enriquecimiento de bosque en los

Tabla 2. Especies forestales asociadas con el carrá en la zona de estudio

\begin{tabular}{|c|c|c|}
\hline Nombre vulgar & Nombre científico & Familia \\
\hline Lechero & Brosimum utile & Moraceae \\
\hline Yurumo & Cecropia sp. & Cecropiaceae \\
\hline Cuangare & Dialyanthera gracilipes A.C & Myristicaceae \\
\hline Caimito & Micropholis sp. & Sapotaceae \\
\hline Palma barrigona & Dictyocaryum platysepalum & Arecaceae \\
\hline Hobo & Sponbdas mombin $\mathrm{L}$. & Anacardiaceae \\
\hline Jagua & Genipa americana & Rubeaceae \\
\hline Tachuelo & Solanun cf. Inopinum & Solanaceae \\
\hline Guácimo & Guazuma olmifolia & Esterculiaceae \\
\hline Pinguaci & Schizolobium parahybum & Caesalpinaceae \\
\hline Hormigo & Lunania parviflora & Flacourtiaceae \\
\hline Guamo & Inga $s p$ & Mimosaceae \\
\hline Costillo & Sagotia racemosa & Euphorbiaceae \\
\hline Laurel & Laurus nobilis L & Lauráceas \\
\hline Aserrín & Parkia pendula & Mimosaceae \\
\hline Guina & Carapa guianensis & Meliaceae \\
\hline Caucho & Castilloa elastica Sesé, C. Tunu & Moraceae \\
\hline Sangre gallina & Vismia macrophylla & Hypericaceae \\
\hline
\end{tabular}




\section{Carrá en zona alta y media del río Baudó. HL Moreno Mosquera}

sitios donde se evidencian claros reportes de escasez de la especie $H$. patinoi.

En cuanto a la calidad del fuste, se considera que la especie muestra un buen comportamiento en estado natural, pues el reporte de árboles con mal formaciones (partidos y bifurcados) fue bajo; sin embargo, se recomienda la implementación de algunas labores de manejo silvicultural, para reducir a la mínima expresión este porcentaje.

\section{Agradecimientos}

El autor expresa sus más sinceros agradecimientos al Instituto de Investigaciones Ambientales del Pacífico (IIAP), director ingeniero William Klinger por la dirección del trabajo, a Eyda Annier Moreno por su asesoría, a consejos comunitarios de Almendro, Bellavista, Berreberre, Puerto Echeverri, Bellavista, Dubasa, Puerto Aracely y Puerto Córdoba en los municipios de Alto y Medio Baudó.

\section{Literatura citada}

Cárdenas D, Salinas NR (eds.). 2007. Libro rojo de plantas de Colombia. Volumen 4. Especies maderables amenazadas. Primera parte. Serie libros rojos de especies amenazadas de Colombia. Bogotá: Instituto Amazónico de Investigaciones Científicas (SINCHI), Ministerio de Ambiente, Vivienda y Desarrollo Territorial; 234 pp. URL disponible en: https://www.sinchi.org.co/ files/publicaciones/publicaciones/pdf/LR_MADERABLES.pdf

Corporación Autónoma Regional para el Desarrollo Sostenible del Chocó. Corporación Montaña. 2010. Plan de ordenación forestal para la cuenca Media y Baja del río Baudó. Informe técnico. Quibdó: CODECHOCÓ; 119 pp.

García H. 2012. Deforestación en Colombia. Retos y perspectivas. Bogotá: Fedesarrollo. URL disponible en: http://www.repository.fedesarrollo.org.co/handle/11445/337

Guariguata MR. 1998. Consideraciones ecológicas sobre la regeneración natural aplicada al manejo forestal. Serie Técnica. Informe Técnico $\mathrm{N}^{\circ}$ 304. Turrialba: CATIE; 27 pp.

Instituto de Investigaciones Ambientales del Pacífico(IIAP) y Corporación Autónoma para el Desarrollo Sostenible del Chocó (CODECHOCÓ). 2008. Convenio $N^{\circ} 005$ interadministrativo, el proyecto Implementación de estudio base para especies forestales amenazadas en el departamento del Chocó. Quibdó: IIAP, CODECHOCÓ. URL disponible en: http://siatpc.iiap.org.co/ docs/avances/lbefa.pdf

Lamprecht H. 1990. Silvicultura en los trópicos: los ecosistemas en los bosques tropicales y sus especies arbóreas. Posibilidades para un aprovechamiento sostenido. Roma: FAO.

Mosquera Ramos LJ, Robledo Murillo D, Asprilla Palacios A. 2007. Diversidad florística de dos zonas de bosque tropical húmedo en el municipio de Alto Baudó, Chocó, Colombia. Acta Biol Colomb. 12: 75-90. URL disponible en: https://revistas.unal.edu.co/index.php/ actabiol/article/view/27244

Murillo D. 2009. Implementación de estudio base para especies forestales amenazadas, en el municipio de Alto Baudó, departamento del Chocó, Colombia. Bioetnia. 6 (2): 82-92. URL disponible en: http://iiap.org.co/ documents/a31294024ba801858dc172b6f72eabb5. pdf\#page $=4$

Rengifo-Ibargüen R. 2010. Estado de conservación de tres plantas útiles del municipio de Lloró, Chocó, Colombia. Revista Institucional Universidad Tecnológica del Chocó Investigación Biodiversidad y Desarrollo. 29 (1):25-34. URL disponible en: https://revistas. utch.edu.co/ojs5/index.php/revinvestigacion/article/ view/310/299 